\title{
Study on the performance of MOS - based displacement sensor
}

\author{
Baoshan Wang ${ }^{1}$, Lijie Cao*2 \\ ${ }^{1}$ College of Mechanical \& Automotive Engineering, Shanghai University of Engineering Science, Shanghai, China \\ ${ }^{2}$ College of Mechanical \& Automotive Engineering, Shanghai University of Engineering Science, Shanghai, China
}

\begin{abstract}
The AZO film has a laser-induced lateral photovoltage (LPV): the saturation values of this photovoltage vary linearly with the position of laser spot. While, we coated a diatomite film on position sensitive detectors based on $\mathrm{AZO}-\mathrm{SiO}_{2}-\mathrm{Si}$ structure. Sensitivity and saturation power are apparently improved, and the sensitivity reached $108.1 \mathrm{mw} / \mathrm{mm}$ for the transient peak value. Maximum saturation voltage reached $72.1 \mathrm{mw}$. This work demonstrates a novel way to increase sensitivity for position sensitive detectors.
\end{abstract}

\section{Introduction}

Lateral photovoltaic effect (LPE) was first discovered by Schottky in 1930, and then expanded by Wallmark in $1957[1,2]$. It has been used in varieties of sensors, such as position sensitive detectors, due to its output of lateral photovoltaic (LPV) changing with the laser position linearly. In the early $21 \mathrm{st}$ century, Henry and Livingstone of Australia systematically studied the LPE of various metal semiconductor junctions. LPE is mainly studied in terms of position, wavelength, temperature, illumination, power and bias. In the past, researchers mainly tried to improve the position sensitivity for practical applications. However, the reported sensitivities of LPV in some MOS and MS structures were mostly within the range of $0.5-100 \mathrm{mV} / \mathrm{mm}$, which can't meet the requirement of high precision PSDs. In order to improve the sensitivity of device, LPE was mainly studied in aspect of laser wavelength, experimental temperature, lateral illumination, laser power and bias voltage. Afterwards, sensitivity, linearity, and saturation photovoltage were significantly improved by coating with a carbon film and graphene sheet (GS) on PSDs based on the MOS structures. To further investigate LPE, we have reported a PSD based on AZO$\mathrm{SiO}_{2}-\mathrm{Si}$ structure with large sensitivity and good linearity for the first time, and it can meet the requirement of high precision PSDs.

To further investigate LPE, in this paper, we reported a position sensitive detector (PSD) based on AZO-SiO - -Si structure with a large LPV sensitivity and a good linearity [3 6]. AZO, the forbidden band width is generally greater than $4 \mathrm{eV}$ and changing with its components. It has resistivity of $20-30 \Omega \bullet \mathrm{cm}$ magnitude and transmittance of visible light is $60 \% \sim 70 \%$. The thermal stability of temperature is above $400{ }^{\circ} \mathrm{C}$. The short circuit current and conversion efficiency of solar cells can be greatly improved by applying to non- crystalline silicon solar cells. They are considered as good support for catalysts and adsorbents. Due to improve the sensitivity, linearity and saturation photovoltage of LPE, the AZO surface was coated with diatomite. As a result, we achieved high precision of measurement and improved sensitivity of PSD [7].

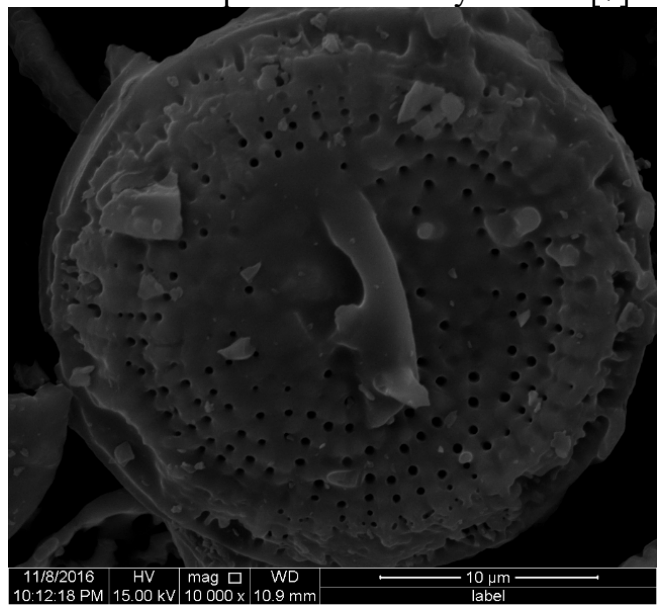

Figure 1. Microstructure diagram of the diatomite

\section{Experimental details}

Figure.1 shows the SEM image of the diatomite, which was measured by SEM. This image reveals the microstructure of the diatomite. The pore size of the diatomite was observed to be between 50 and $3000 \mathrm{~nm}$, while the pore size of diatomite the size of the electron interference was met, and the enhanced (LPE) could be obtained on the conductive layer of Diatomite-AZO$\mathrm{SiO}_{2}-\mathrm{Si}$ structure. It presents the optical transmittance spectra of a $100 \mathrm{~nm}$ thick AZO film corrected for the attenuation of a glass substrate. The film is highly transparent in the Vis-IR region with a transmittance between $60 \%$ and $90 \%$. It shows that AZO film is a good transparent conducting oxide in Vis-IR region. As we

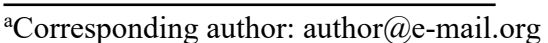


can see, all the samples output satisfy sensitivity and show good linearity. All data and analyses demonstrate that AZO may be a candidate for PSDs [8].

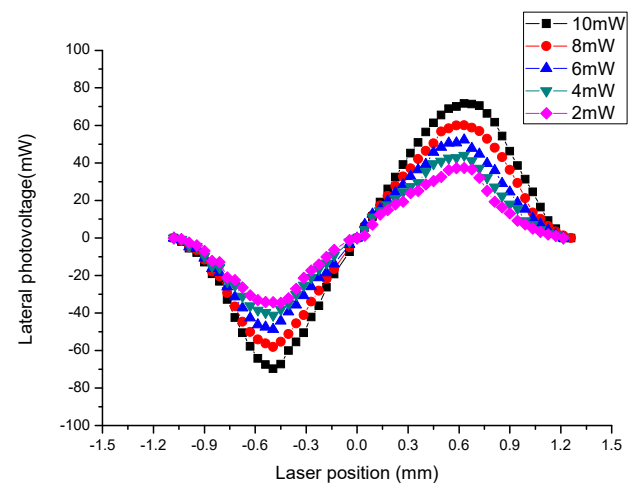

Figure 3. LPV under the illumination of different laser power (512 $\mathrm{nm}$ wavelength).

\section{Experimental procedure}

Samples were fabricated on n-type silicon with thickness of $0.3 \mathrm{~mm}$. These samples were cut into $15 \mathrm{~mm} \times 35 \mathrm{~mm}$ rectangles. Because silicon is oxidized in the air, the surface naturally forms a $\mathrm{SiO}_{2}$ layer of about $1 \mathrm{~nm}$ thick. An AZO film was formed on oxidized silicon substrate by the method of spin coating, and a sample with AZO$\mathrm{SiO}_{2}$-Si metal oxide-oxide-semiconductor material was fabricated after the film was vacuum dried. The silicon substrate is immersed in the solution; the beaker is put into the ultrasonic cleaning machine. The larger AZO powder can be dispersed by ultrasonic oscillation of 20 min. We take it out of the beaker after the base plate is soaked for two hours. Then the glue is used for $3 \mathrm{~min}$ at the speed of 5, $000 \mathrm{r} \bullet \mathrm{min}-1$. AZO particles are evenly distributed on the substrate. Remove the basal piece into the dryer with $75{ }^{\circ} \mathrm{C}$ for two hours. As a result, we obtained a $\mathrm{AZO}-\mathrm{SiO}_{2}-\mathrm{Si}$ substance which is firmly attached to AZO films.

We made a $2 \mathrm{~nm}$ diatomite layer on the AZO films by means of the vacuum deposition by spin coating. It leads to a diatomite-AZO-SiO2-Si structure. To investigate LPE, the samples were scanned spatially with a visible laser ( $9 \mathrm{~mW}$ and $512 \mathrm{~nm}$ ) focused on a roughly $50-\mu \mathrm{m}$ diameter spot at the AZO film surface without any spurious illumination (e.g. background light) reaching the samples.

The schematic set-up for the LPV measurement is shown in Figure 2. Both alloying indium contacts (less than $1 \mathrm{~mm}$ in diameter) were attached to the bottom of the Si substrate. The distance between the two indium electrodes is $2.25 \mathrm{~mm}$. The curves of the twodimensional lateral photovoltaic ( LPV) were plotted . Data modeling by Origin8.0 displayed experimental results in pictures after the laser scanning was done .

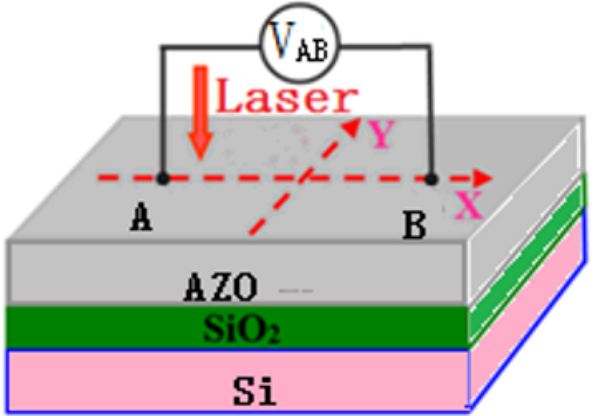

Figure 2.Geometry of the MOS structure

\section{Results and discussion}

As the Figure 3 shows, the maximum value of the voltage appears at the two electrode points, and the voltage - position function relation curve between the two electrodes is approximately linearly related at different power conditions, and the voltage is approximately $0 \mathrm{mV}$ when the laser light just irradiates the center position of the two electrodes. The laser powers used to illuminate the surface are $2 \mathrm{~mW}, 4 \mathrm{~mW}$, $6 \mathrm{~mW}, 8 \mathrm{~mW}$ and $10 \mathrm{~mW}$ respectively. Figure 4 shows the experimental results of the induced LPV as a function of the laser spot position for $\mathrm{AZO}-\mathrm{SiO}_{2}-\mathrm{Si}$ structure.

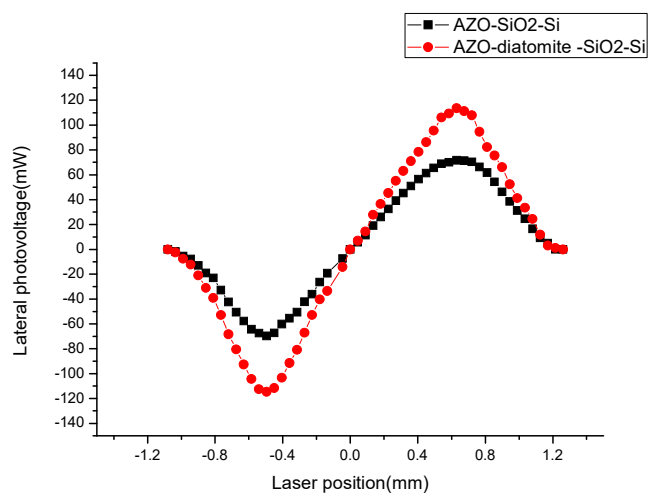

Figure $4 \mathrm{LPV}$ under $\mathrm{AZO}-\mathrm{SiO}_{2}$-Si structure, diatomite-AZO$\mathrm{SiO}_{2}-\mathrm{Si}$ structure

To explain the LPE observed in AZO film based on $\mathrm{AZO}-\mathrm{SiO}_{2}-\mathrm{Si}$ homo-heterostructure, we propose the following physical model. Figure 5(a) shows the energy band diagram of the $\mathrm{AZO}-\mathrm{SiO}_{2}-\mathrm{Si}$ system in equilibrium state. A barrier is formed to integrate the two Fermi levels by energy band bending. The oxide layer has a tunneling thickness (about $1.2 \mathrm{~nm}$ ) according to extensive works. When AZO film is illuminated by the $512 \mathrm{~nm}$ laser, energy is mainly absorbed by $\mathrm{Si}$ substrate where generating electron-hole pairs. The generated electrons tunnel through $\mathrm{SiO}_{2}$ into $\mathrm{AZO}$ layer while the holes are left in $\mathrm{Si}$ substrate. These excess carriers generate a concentration gradient between the illuminated spot and nun-illuminated zone. Due to the concentration gradient these excess carriers move laterally away from the 
illuminated spot. Noticeable factor is AZO film is quite thin and must be described by surface concentration. As a result, with the same number carrier injected, AZO film concentration is much more influenced [9].

Under laser irradiation, the band structure of AZODiatomite-SiO2-Si structure in equilibrium state is approximately similar to that of AZO-SiO2-Si structure, and also has tunneling layer, depletion layer and neutral layer. The electrons of the conduction band acquire the laser energy, then escape from the n-Si semiconductor, and then go to the Diatomite layer after the oxide layer passes through. In this process, due to the built-in electric field and the direction of electron transition in the same direction, and the electron tunneling interface will be affected by the loss of energy, with the increase of electric field intensity and electron energy decreases, the electronic excitation light will not escape, the electronic system and the corresponding escape hole reaches the equilibrium state [10]. Due to the diffusion of the excess electrons, two quasi-Fermi levels were produced at the contact electrode as shown in Figure 5(b).Under laser irradiation; the band diagram of AZO$\mathrm{SiO} 2-\mathrm{Si}$ structure in equilibrium state is similar to AZOdiatomite-SiO2-Si.

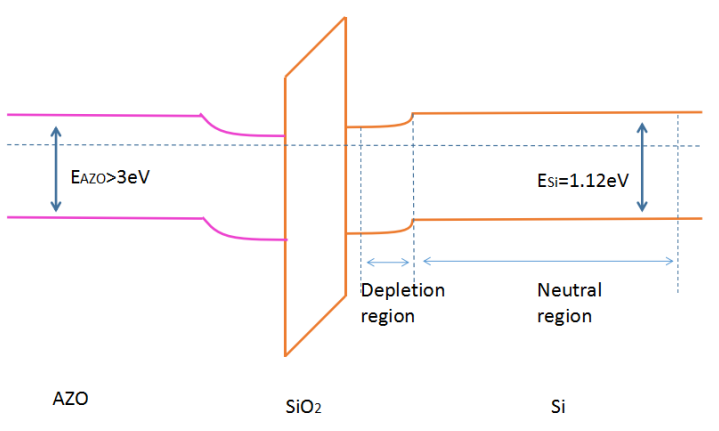

(a)

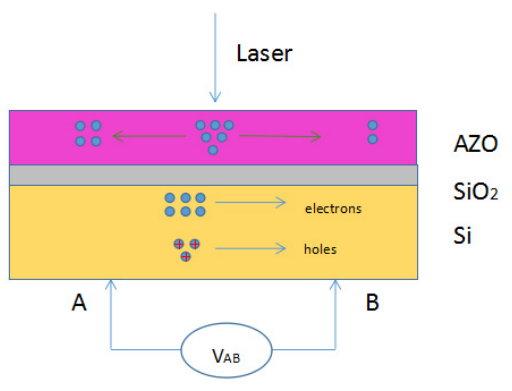

(b)

Figure 5(a). schematic simple equilibrium energy-band diagram of AZO-SiO2-Si,(b)schematic profile diagram of the excess carrier diffusion on AZO surface under a spot illumination

Due to coating a diatomite thin film on $\mathrm{AZO}-\mathrm{SiO}_{2}-\mathrm{Si}$ structure. Sensitivity and saturation power are apparently improved. For the better investigation, an ideal onedimensional model is given by the quantitative explanation. For AZO-diatomite- $\mathrm{SiO}_{2}-\mathrm{Si}$ structure , when the pore size of the diatomite structure matches the electron matter wavelength, the electron will inevitably interfere in the conductive layer. It can lead to homologous changes of voltage.

When the surface of sample is irradiated by laser, the electrons produced by the excitation of the $\mathrm{Si}$ semiconductor diffuse through the $\mathrm{SiO}_{2}$ layer to the AZO layer. Due to the electron interference effect in each of the pores of the diatomite, several electron enhancement points are formed in the AZO conductive layer. Due to the different distance between the electron enhancement point and the laser point, the electron density of the electron enhancement point in each position of the electrode is different. Therefore, when these electrons diffuse to the two electrodes, the enhanced lateral photovoltaic effect is produced due to the difference of diffusion distance and electron density [11 14].

\section{Conclusions}

In summary, the AZO film was first found to exhibit a laser-induced lateral photovoltaic effect. When the laser spot scans along the line between the two electrodes, we can observe a linear increase in the voltage with the distance between the laser spot and the center position between the two electrodes. Comparing to the AZO$\mathrm{SiO}_{2}-\mathrm{Si}$ structure and diatomite-AZO-SiO $2-\mathrm{Si}$, Due to the interference effect of porous film, the LPV and sensitivity in the diatomite- $\mathrm{AZO}-\mathrm{SiO}_{2}-\mathrm{Si}$ device has a maximum increase, the sensitivity become larger. We believe that the diatomite coating can provide a new method to increase LPE based on the MOS structure [15 16]. The linearity is expected to make diatomite$\mathrm{AZO}-\mathrm{SiO}_{2}-\mathrm{Si}$ a new type of candidate for positionsensitive photodetector.

\section{References}

1. Schottky W1930 Phys. Z.31 913 (1930)

2. J. T. Wallmark, IEEE, 45, 4 (1957).

3. J. Henry, J. Livingstone J. Phys. D: Appl. Phys., 34 (2001).

4. I. S. Jeong, J. H. Kim, and S. Im, Appl. Phys. Lett. 83, 14 (2003).

5. T. Dietl, H. Ohno, F. Matsukura, J. Cibert, and D. Ferrand, Science, 287 (2000).

6. R. H. Willens, Appl. Phys. Lett. 49 (1986).

7. S. Liu, X. Xie, H. Wang Optic Express, 22 (2014).

8. J. Henry, and J. Livingstone, J. Mater. Sci. Mater. Electron. 12, 7 (2001).

9. C. Q. Yu, H. Wang, and Y. X. Xia, Appl. Phys. Lett. 95 (2009).

10. B. S. Chun, H. C. Wu, M. Abid, I. C. Chu, S. Serrano-Guisan, I. V. Shvets, and D. S. Choi, Appl. Phys. Lett. 97, 8 (2010)

11. Y. Liu et al., MATER LETT, 161 , 747 (2015).

12. J. Liu et al, MATER LETT, 168, 48 (2016).

13. S. Zhao et al., Optik , 124, 1105 (2013). 
14. V. Zhelev et al, THIN SOLID FILMS, 22, 102 (2018).

15. Cong. RD et al, Adv Sci. 5, 5 (2017).

16. C. Q. Yu et al., Opt. Lett. 34, 3770 (2009). 